\title{
BOOK REVIEW \\ BŘEZINOVÁ, HELENA: SLAVÍCI, MOŘSKÉ VÍLY A BOLAVÉ ZUBY. POHÁDKY H. CH. ANDERSENA: MEZI ROMANTISMEM A MODERNITOU. BRNO, 2019
}

\author{
Martin Liška \\ Karlsuniversität, Prag \\ martin-liska@seznam.cz
}

Niemand kann bestreiten, dass der Erfolg der Märchen von Hans Christian Andersen schon seit Langem die Grenzen Dänemarks überschritten hat. Tschechien stellt in dieser Hinsicht keine Ausnahme dar, und den hiesigen Lesern steht eine Menge von Märchenbüchern, die Andersens Namen tragen, zur Verfügung - warum an dieser Stelle nicht von Andersens Märchenbüchern Sprache ist, wird später in diesem Text klargestellt. Daher ist es wirklich erstaunlich, dass ein eventueller interessierter Leser, der etwas mehr über den Märchenautor und seine Schöpfung erfahren möchte, keine Übersicht der Geschichte der dänischen Literatur auf Tschechisch benutzen kann (im Gegensatz zu der norwegischen und schwedischen Literatur). Lange gab es auf Tschechisch auch weder eine Biographie Andersens, wenn man seine Autobiographie Pohádka mého života (Mit Livs Eventyr, "Das Märchen meines Lebens") nicht als solche betrachten will, noch eine selbständige Studie, die sich seinem Werk widmen würde. Diese Lücke in der tschechischen skandinavistichen Forschung wurde erst neulich geschlossen, und zwar mit dem Werk Slavíci, mořské víly a bolavé zuby. Pohádky H. Ch. Andersena: Mezi romantismem a modernitou ("Nachtigallen, Meerjungfrauen und schmerzende Zähne. Die Märchen von H. Ch. Andersen: Zwischen Romantik und Modernität") von Helena Březinová, das im Herbst 2018 erschienen ist.

Helena Březinová (geb. 1974) ist seit 2002 am Lehrstuhl für skandinavistische Studien der Philosophischen Fakultät der Karlsuniversität in Prag tätig, seit einigen Jahren leitet sie ihn. Fachmännisch hat sie sich auf die dänische Literatur des 19. und 20 Jahrhunderts spezialisiert und mehrmals ein Seminar über das Werk Hans Christian Andersens geleitet, man wird also im Rahmen der tschechischen Skandinavistik und Literaturwissenschaft kaum jemanden finden, der kompetenter wäre, eine Monographie über diesen bedeutenden Schriftsteller zu verfassen.

Die besagte Monographie ist keineswegs als eine Biographie Andersens zu lesen, und es werden nur sehr wenige rein biographische Angaben über ihn im Text angegeben. Die Autorin erinnert den Leser außerdem an mehreren Stellen im Text, dass sie auch nicht beabsichtigt, selbstbiographische Elemente in Andersens Märchen zu identifizieren und zu analysieren. Diesem Vorsatz folgt sie so treu, dass sie erst auf Seite 238 verrät, dass Andersen im Prinzip sein ganzes Leben an Zahnschmerzen gelitten hat, und damit die schmerzenden Zähne im Titel der Monographie erklärt. Březinová konzentriert sich stattdessen auf zwei wesentliche Themen: teilweise auf die Übersetzungen von Andersens Werken ins Tschechische und überwiegend auf die Weise, wie seine Texte zu interpretieren sind. Diese zwei Themen kommen ziemlich oft miteinander in Berührung, denn die Weise, auf die die Übersetzer die Originaltexte lasen und verstanden, beeinflusste oft auch die von ihnen stammende Übersetzung.

Der vorherige Satz gilt allerdings natürlich nur für jene Übersetzer, die sich bemühten, Andersens Texte auf Dänisch zu lesen und der Versuchung, sie zu ändern, zu verbessern oder für Kinder erträglicher zu machen, widerstanden. Diese heute als selbstverständlich betrachteten Grundregeln der Übersetzungskunst wurden und werden leider auch immer noch von tschechischen Übersetzern und Verlegern ignoriert. Im 19. Jahrhundert gehörte es zum allgemein akzeptierten Standard, dass ursprünglich in einer "kleinen" Sprache verfasste Werke aus ihrer deutschen Übersetzung weiter ins Tschechische übersetzt wurden, dass die Handlung oft in ein Milieu versetzt wurde, das den kleinen tschechischen (oder eher böhmischen und 
mährischen) Lesern mehr bekannt war, und dass kürzere oder längere Abschnitte aus dem Text gestrichen wurden. Dass aber sogar nach dem Jahr 2000 Andersens Märchen- und Erzählungssammlungen erschienen, die nicht aus dem Dänischen, sondern aus dem Englischen, Deutschen oder sogar - nur Gott und der Verleger wissen wohl warum - aus dem Ungarischen übersetzt wurden, und die man eher "freie Bearbeitungen von freien Bearbeitungen von Andersens Texten" als "Übersetzungen" nennen sollte, das lässt sich kaum mit Hinweis auf Mangel an kompetenten Übersetzern entschuldigen. Der wirkliche Andersen liegt hinter allen diesen Versionen verborgen und es ist sehr schwer, den richtigen Weg $\mathrm{zu}$ ihm $\mathrm{zu}$ finden. Glücklicherweise gibt hier Březinová dem Leser eine helfende Hand und weist auf jene Übersetzungen hin, die dem Original möglichst nah stehen. Wie aber an einigen Stellen erwähnt wird, scheinen manchmal selbst diese empfohlenen Übersetzungen etwas von der ursprünglichen Bedeutung verloren zu haben, wie Březinovás Interpretationen zeigen. Auf diesem Wege würde man jedoch bald zu den allgemeinen Theorien und Diskussionen kommen, wie treu selbst die beste Übersetzung dem Original sein kann, und damit zu weit von dem eigentlichen Thema des Buches.

Nach dieser Einführung in die Andersen-Problematik im tschechischen Sprachraum und nach einer kurzen Übersicht über die Geschichte des Märchens als selbständigen Genres und dessen theoretischen Gründen und Attributen tritt die Autorin zum Hauptthema des Buches über, nämlich zu Analysen und Interpretationen von Andersens Texten. Vor allem konzentriert sich Březinová auf die Ironie, von der die Märchen durchgedrungen sind, die allerdings nur für den erwachsenen Leser deutlich ist und die der Moral der Märchen einen ganz anderen Sinn gibt als von den Kindern verstanden; auf die Zweideutigkeit, mit der Andersens Texte zu lesen sind; und auf Elemente des Modernismus, die man bei Andersen bereits erkennen kann. Außerdem erklärt Březinová ausführlich den Unterschied zwischen dem neutralen Erzähler einer Geschichte ("narrator" auf Englisch) und einem, der aktiv eine Stellung im oder mit dem Text nimmt; der zweitgenannte ("storyteller" auf Englisch) kommt bei Andersen viel häufiger vor. Vor seiner Ironie und seinen vielen Schelmenstücken muss sich der Leser oft in Acht nehmen, sonst kann er die Moral der Geschichte oder sogar die Handlung selbst völlig missverstehen Březinová selber schreibt mehrmals, dass uns der Erzähler mehr oder weniger deutlich anblinzelt und uns damit warnt, dass wir uns nicht allzu sehr auf das geschriebene Wort verlassen sollen. So interpretiert sie zum Beispiel Ole Zavřiočka (Ole Lukøie, "Ole Luk-oie") als verkappten Antibildungsroman und macht auf die Ironie aufmerksam, vor der Slavík (Nattergalen, "Die Nachtigall") strotzt. Einen großen Raum widmet Březinová der Frage, inwiefern einige Texte von Andersen als Parabeln bezeichnet werden können. Dieses Genre wird oft mit Franz Kafka verbunden, Březinovás Analysen zeigen aber deutlich, dass man auch bei Andersen in vielen Fällen über Parabeln sprechen kann, obwohl nicht völlig klar ist, ob er selber seine Texte als Parabeln wahrnahm oder nicht. In diesem Zusammenhang muss erwähnt werden, dass Březinová deutlich hervorhebt, dass es nicht darum geht, ob Kafka oder Andersen der erste war, der Parabeln in der modernistischen Literatur vorstellte. Genauso sachlich und unspektakulär erwähnt sie, dass Andersens Texte Kafka offenbar bekannt waren, ohne den eventuellen Einfluss Andersens auf Kafkas Werk zu übertreiben. Zu Franz Kafka kehrt sie nochmals in dem Kapitel zurück, wo sie Andersens Erzählung Stín (Skyggen, "Der Schatten") und Kafkas Text Ein Hungerkünstler vergleicht. Obwohl diese Texte sowohl in der äußeren Form als auch in der Handlung sehr unterschiedlich sind, kommt Březinová zu der Schlussfolgerung, dass sie einander ähneln, dass man bei näherer Betrachtung nicht eindeutig entscheiden kann, welche der handelnden Personen der Gute und welche der Böse ist, obwohl dies auf den ersten Blick ganz klar zu sein scheint.

Besondere Aufmerksamkeit widmet Březinová der Sprache und der versagenden Kommunikationsweise zwischen einzelnen Figuren in einigen Märchen. Neben einigen, eher weniger bedeutenden, Aspekten dieser Problematik, wie zum Beispiel die Rolle von Duzen und 
Siezen zwischen dem Künstler und seinem Schatten in Stín, konzentriert sich Březinová auf detaillierte Analysen der Märchen Statečný cinový vojáček (Den standhaftige Tinsoldat, "Der standhafte Zinnsoldat"), Pastýrka a kominíček (Hyrdinden og Skorsteeinsfeieren, "Die Hirtin und der Schornsteinfeger"), Smrček (Grantræet, "Der Tannenbaum") und natürlich am detailliertesten auf die Malá mořská vila (Den lille Havfrue, "Die kleine Meerjungfrau"), in der das Sprachproblem dank des Motivs der abgeschnittenen Zunge am deutlichsten hervortritt. Březinová findet verschiedene Erklärungen dafür, warum die Figuren einander manchmal sogar fatal missverstehen, wobei die Unzufriedenheit Andersens mit der Tatsache, dass das Publikum seine Texte teilweise oder völlig falsch interpretiert, eine wichtige Rolle spielt.

Ohne Bemerkung ließ Březinová auch nicht das Verhältnis zwischen Hans Christian Andersen und einem seiner berühmtesten dänischen Zeitgenossen, Søren Kierkegaard. Eines der allerletzten Märchen Andersens, Teta Bolízubka (Tante Tandpine, "Tante Zahnweh"), interpretiert sie nicht nur als Andersens schöpferisches Testament, sondern auch als eine offene Polemik mit Kierkegaard und mit Georg Brandes über die Bedeutung und Aufgabe der Literatur.

Der dritte Teil von Březinovás Buch besteht aus neuen Übersetzungen von drei weniger bekannten Andersen-Märchen, nämlich Teta Bolizubka (Tante Tandpine, "Tante Zahnweh"), "Ve městě jsou bludičky, " pravila bába z bažiny (Lygtemændene ere i Byen, sagde Mosekonen, "Die Irrlichter sind in der Stadt, sagte die Moorfrau") und Zelení drobečkové (De smaa Grønne, "Die kleinen Grünen"). Alle drei wurden auf Tschechisch zum letzten Mal in einer Ausgabe zwischen 1914 und 1916 veröffentlicht, und deswegen war es an der Zeit, sie den tschechischen Lesern wieder verfügbar zu machen, vor allem da sich die Autorin in ihrem Text oft auf sie bezieht. Diese Übersetzungen sind ebenfalls das Werk von Helena Březinová.

Auch wenn, wie oben erwähnt, Březinovás Werk die erste Monographie über Andersen auf Tschechisch ist, handelt es sich keinesfalls um eine einfache Einleitung in das Thema, sondern um einen wichtigen Beitrag zur Andersen-Forschung, der auch die strengen Kriterien eines internationalen Vergleichs erfüllt. Březinová baut ihre Interpretationen und Schlussfolgerungen auf einer soliden Basis von Quellen- und Literaturhinweisen auf, wobei sie natürlich vor allem dänische, aber auch deutsche und englische Forscher zitiert. Natürlich muss man mit ihren Behauptungen nicht immer einverstanden sein (zum Beispiel basiert ihre sechsseitige Interpretation des Märchens Srdce žal (Hjertesorg, "Ein Herzeleid") im Prinzip auf dem wenig verbreiteten dänischen Wort "forkundskaber", das Andersen in der Einleitung des Märchens benutzte, wobei sie einen direkten Zusammenhang mit zwei seiner Briefe sieht, wo er dasselbe Wort verwendete), sie findet aber immer logische Argumente, die ihre Hypothese unterstützen. Ein aufmerksamer Leser stößt im Text sogar auf Stellen, wo ihre Sprache absichtlich oder nicht die von Andersen imitiert: "Ted už je ale opravdu nejvyššsi čas nastínit děj" ("Jetzt ist es aber wirklich höchste Zeit, über die Handlung zu berichten", S. 217). Hiermit hängt auch das konsequente Benutzen des Plurals zusammen, wenn die Autorin über sich und ihr Buch spricht - immer ist die Rede von "unserem Buch", über das, was "wir sehen" usw., bis sie sogar den Leser in die Entscheidung miteinbezieht, dass die drei neuen Übersetzungen in das Buch eingegliedert werden: "Rozhodli jsem se tuto pohádku uvést v naši knize v novém překladu" ("Wir haben uns entschieden, dieses Märchen in unserem Buch in einer neuen Übersetzung anzugeben", S. 215). Es befinden sich im Text auch Stellen, wo sie mit einer Ironie im wahren Geiste Andersens zugibt, dass die Forschung wahrscheinlich niemals völlig klarstellen wird, was für Belehrung Andersen den Lesern zu erteilen beabsichtigte.

Und mit einer ironischen Tatsache kann man diese Rezension beenden: Das Buch, in dem seine Autorin mit allen ihren Kräften, Fähigkeiten und Kompetenzen den Leser zu überzeugen versucht, dass Andersens Märchen sich nicht (nur) an Kinder richten, wurde 2019 mit dem ersten Preis im tschechischen Literaturwettbewerb Zlatá stuha ("Die goldene Schleife") belohnt, und zwar in der Kategorie "Theorie und Kritik der Literatur für Kinder und Jugendliche". 